\title{
Planeación urbana, participación ciudadana y cambio social 1
}

\author{
Boris Graizbord \\ El Colegio de México/LEAD-México
}

\section{Introducción}

La idea de participación ciudadana o pública no es nueva. En Inglaterra se institucionalizó como parte de los cambios que sufrió el proceso de planificación a partir del reporte "Skeffington" a fines de la década de los sesenta. El antecedente inmediato era la ley de planeación de 1968, que constituyó en la Gran Bretaña un hito en el desarrollo de un marco de planeación con un alto grado de participación ciudadana. Sin embargo, el estímulo venía del gobierno central, pues era necesario que los gobiernos locales se organizaran apropiadamente para ejercer sus funciones de planificación.

El gobierno británico convocó en marzo de 1968 a un comité bajo la dirección del que era secretario del ministro de Vivienda y Gobierno Local, Arthur Skeffington, quien publicó el reporte "People \& Planning" en 1969. Las recomendaciones parecían obvias:

${ }^{1}$ Ponencia elaborada para el Encuentro Internacional sobre Participación en la Gestión del Medio Ambiente, organizado por la Secretaría del Medio Ambiente del Gobierno del Distrito Federal y el Centro Internacional para el Desarrollo (CIID/IDRC-Canadá), celebrado en la ciudad de México los días 16 al 18 de noviembre de 1998.

Economía, Sociedad y Territorio, vol. II, núm. 5, 1999, 149-161. 
- la población debía mantenerse informada acerca de la preparación de los planes locales de sus correspondientes jurisdicciones político-administrativas;

- las autoridades locales de planeación debían publicar propuestas de forma que quedaran claras sus implicaciones para los residentes en el área a la que se refería el plan;

- el público debía ser notificado de los logros de sus representantes sobre lo que habían aceptado y por qué lo habían hecho; y

- la gente debía ser animada a participar en la preparación de los planes, no sólo haciendo comentarios, sino también involucrándose en encuestas y otras actividades relacionadas con el proceso de planeación.

Entre estas recomendaciones y la realidad había, por supuesto, una enorme distancia: el cómo. En efecto, la brecha entre los procedimientos de planeación y los intereses más amplios de la ciudadanía, entre planeación física y política económica y social, entre las propuestas y sus implicaciones políticas, sólo podía cerrarse con una transferencia de poder que permitiera decidir aquellas cuestiones de interés para la población local. En ese momento se hizo evidente que:

1. Había que ir más allá de los procedimientos formales, las encuestas y las exhibiciones públicas de las propuestas y los planes.

2. El proceso era político y debía rebasar la planeación urbana.

3. Había que encontrar y construir vínculos entre participación popular y democracia representativa.

4. La participación debía ser un medio para que los consumidores y usuarios controlaran el poder burocrático y profesional de los planificadores o tomadores de decisiones.

5. La representatividad social de los ciudadanos organizados y participantes era limitada: no cubría a toda la población y no se refería a todos los problemas o intereses existentes en un lugar y momento dados.

6. Existía la posibilidad de que la participación ciudadana llevara a propuestas excluyentes o que implicara enormes costos sociales que afectarían a una comunidad más amplia o a la ciudadanía en su conjunto. ${ }^{2}$

${ }^{2}$ Esto es especialmente válido cuando la participación de la población se da de manera desinformada o sin responsabilidad. Tudela (citado en Tamayo, 1998) lo relaciona con la inercia que los paradigmas convencionales llevan consigo, perpetuando 
7. Era necesario conciliar lo que debía mantenerse en secreto para evitar su difusión y, por tanto, su uso especulativo antes de tiempo, y el derecho a ser informado o el deber de informar, lo cual resultaba difícil ante una mayor participación ciudadana.

Considerando los antecedentes anteriores, en estas notas se propone un modelo de análisis de la participación -o, más bien, una interpretación de la misma como proceso ${ }^{3}$ de planeación y cambio social- y se señalan algunas de las enormes dificultades que implica la participación de la población y de las condiciones para su efectividad en la escala metropolitana para el caso de la ciudad de México, o de una ciudad tan grande como ésta. Divido el texto en una introducción, la propuesta del modelo y algunos aspectos del mismo; hago referencia en un tercer apartado a la población desorganizada y, finalmente presento unas conclusiones.

\section{Un modelo analítico de participación ciudadana}

Me parece que una efectiva participación ciudadana debe atender cuatro dimensiones:

1. La escala. Seguramente hay diferencias si se trata de ciudad o territorio social y público; o bien de zonas y barrios o territorio comunitario; o, finalmente, de los espacios interaccionales; es decir, del lugar de trabajo, las áreas recreativas, los espacios de uso común vecinales, etcétera.

2. El tiempo. No es lo mismo intervenir ex-post que ex-ante en el proceso de planeación. ${ }^{4}$ Lo primero implica la continua evaluación de resultados que deben convertirse en insumos para la formulación de políticas o identificación de problemas.

3. El contexto. Habría que distinguir entre una situación de rutina y una de crisis. La primera se refiere a procedimientos operativos establecidos, normales, estandarizados, o bien a una

soluciones técnicas aplicables a ciertos casos, pero no necesariamente a otros, sin “[...] asimilar la existencia de opciones diferentes, y sin comprender las principales implicaciones sociales y ambientales de cada uno de ellos", llegando a "demandar sólo lo que se conoce" y no lo que es apropiado. Esto, por supuesto, se aplica a las decisiones de política pública debido tanto a ignorancia como a irresponsabilidad.

${ }^{3}$ C. Tilly, citado en Tamayo, 1997.

${ }^{4}$ Véase Graizbord (1990), para una discusión sobre la relación entre evaluación de impacto ex-post y consideraciones ex-ante en el proceso de planeación y elaboración de políticas. 
estructura establecida -statu quo- que se deriva o refleja valores, normas, intereses, identidades y creencias. Una situación de crisis, por otro lado, implica grandes cambios de política o modificación del espacio político en el que se tiene que tomar decisiones y responder a cambios abruptos en el ambiente institucional.

4. Los motivos de los actores. Es diferente cuando se trata de intereses utilitarios, pecuniarios y materiales, que cuando se tienen motivaciones simbólicas, no pecuniarias y espirituales. Asimismo, no es igual cuando lo que se busca es un fin político que cuando se trata de uno profesional; $y$, finalmente, la intervención puede tener un alcance o motivación inmediatos o bien alentar expectativas en una perspectiva de largo plazo.

En lo que sigue haré referencia a sólo algunos aspectos de cada una de las anteriores dimensiones.

\section{La escala}

En términos de escala, los mecanismos o instituciones ${ }^{5}$ y aspectos en discusión varían según estemos pensando en una decisión que afecta a la toda la ciudad; trátese de un sistema de vialidad y transporte público, del abastecimiento y distribución de agua para la ciudad, o del manejo y operación global de residuos domésticos o industriales.

Es obvio que en esta escala es necesario referirse al "habitante metropolitano", pero en una ciudad concurren diversos agentes e intereses que pueden, desde muy diversos ángulos, percibir las posibilidades de verse afectados o tienen distintas capacidades de articular sus intereses. En esta escala se producen costos sociales directos e indirectos (externalidades) que afectan de alguna manera a unos más que a otros, y deberán crearse mecanismos de transferencia extra-mercado ${ }^{6}$ que subsanen, compensen o mitiguen dichos costos o beneficios no merecidos. Los procesos de decisión no pueden darse a partir de la intervención de un sólo grupo o un agente en particular, y el mecanismo de par-

\footnotetext{
${ }^{5}$ Una institución puede entenderse de diversas maneras: como una regla significativa (derecho de propiedad), una práctica social, pública o privada (toma de decisiones democráticas, herencia, casamiento, familia), un arreglo (niveles de gobierno) o una organización (la universidad, la institución planificadora).

${ }^{6}$ Véanse las críticas al modelo pigouviano de transacción vía el mecanismo del mercado en Bowers, 1997.
} 
ticipación tiene que prever y permitir la expresión de la ciudadanía, de todos los usuarios, de la población en general, etcétera.

En el espacio comunitario y vecinal, por el contrario, los participantes tienen "nombre y apellido", y los mecanismos y decisiones se negocian o consensan ad hoc. Los intereses están representados o tienen voz, ${ }^{7}$ y los procedimientos pueden ser de carácter extraordinario o permanente. El papel que juega aquí el "experto" es relativamente débil, al contrario que en el caso anterior, pero pudiera actuar en calidad de consejero o facilitador (por ejemplo, organizando talleres participativos); es decir, un advocacy planner. ${ }^{8}$

Un ejemplo concreto de participación en el espacio comunitario y vecinal lo ofrece la negociación o "convenio de concertación democrática” que se estableció para el Programa de Renovación Habitacional, que permitió la construcción o rehabilitación de cerca de 50000 viviendas en el Distrito Federal después del sismo de 1985. En este caso las autoridades encargadas, en una situación de crisis, lograron establecer una comunicación positiva con más de 100 grupos de ciudadanos y de población afectada que se organizó e hizo representar en el proceso, diversas organizaciones no lucrativas o no gubernamentales y numerosas empresas del sector privado, si bien se mantuvo una estructura vertical en la integración del proceso (organismos del gobierno federal-Departamento del Distrito Federal-Banco Mundial), característica del funcionamiento de las instituciones políticas y organismos públicos en nuestro país.

\section{El tiempo}

La dimensión temporal, o el momento oportuno y adecuado para auspiciar, aceptar, o propiciar la participación, es un tema tratado en el mencionado reporte "Skeffington". Habría que ver si la participación del público debe darse desde el inicio del proceso, incluso desde la definición del problema, o bien si sólo una vez formulada la propuesta o el plan ésta quedaría al alcance de quien

\footnotetext{
${ }^{7}$ Una explicación de las opciones "salida" y "voz" que tienen los individuos ante un desempeño deliberado de las diferentes organizaciones que producen bienes o servicios, se encuentra en Hirschman, 1970. La "voz", como intermediación o interlocución, es propuesta de manera inteligente en términos de proceso para constituir demandas y de fortalecimiento ciudadano por de la Peña, 1998.

${ }^{8}$ Véase Davidoff (1965), entre otros autores que se refirieron a este grupo de planificadores activistas que surgieron en la década de los sesenta durante el malestar social de esos tiempos en los Estados Unidos.
} 
quisiera revisarla y cualquiera tendría derecho a expresar su opinión al respecto, para modificarla, rechazarla o buscar la anulación parcial o completa de la misma.

La pregunta aquí, como se la hicieron los críticos de aquel reporte, es: ¿quiénes son los interesados en revisar esta propuesta y quiénes tienen la capacidad para entenderla y/o formular una opinión informada al respecto?

\section{El contexto}

La identificación de problemas no es una cuestión trivial, y merece consideraciones no sólo epistemológicas sino también contextuales. Me referiré a estas últimas. Algunos señalan que en estos tiempos, aun sin aceptar que se vive una situación de riesgo globalizado, estamos ante una crisis general y permanente; más aún cuando nuestras grandes urbes alcanzan poblaciones millonarias y extensiones de miles de kilómetros cuadrados. En todo caso, las mega-ciudades, como la ciudad de México, no sólo presentan conocidos riesgos magnificados, sino también nuevas pautas de conflicto político, económico, social y cultural antes no vistas o no consideradas. ${ }^{9}$

Quisiera enumerar algunos de los riesgos y las nuevas pautas conflictivas relacionados con la política urbana y el proceso de toma de decisiones:

1. En la actualidad, no sólo en las ciudades primarias o capitales, sino también en todas las grandes ciudades, se aprecia un incremento considerable en el número de participantes en las decisiones de política urbana: burócratas, grupos vecinales, sindicatos, empleados de servicios públicos, etcétera.

2. Existen innumerables conflictos de índole diversa entre y dentro de las zonas urbanas o barrios en que se divide la ciudad.

3. Proliferan funciones y organismos públicos encargados de éstas tanto en el gobierno de la ciudad como en los de los municipios contiguos que integran la metrópoli.

4. Crece el número de problemas y éstos se politizan y entran en la agenda del gobierno de la ciudad.

5. La fragmentación burocrática y espacial crea conflictos permanentes. ${ }^{10}$

${ }^{9}$ Véase Yeates, 1977.

${ }^{10}$ Véase Graizbord, 1989, para las implicaciones espaciales de la fragmentación política en la ZMCM. 
6. Ha crecido la desconfianza entre ciudadanos y funcionarios públicos, o entre gobernantes y gobernados.

7. Los niveles superiores de gobierno intervienen cada vez más en asuntos urbanos y locales, en parte debido a la importancia y escala de estos problemas.

8. Predominan los problemas sociales y económicos sobre los problemas físicos.

9. Crece la expectativa de que el gobierno puede resolver los problemas, aunada a la percepción de que aquél es el único responsable de éstos.

10. Hay mayor conciencia ciudadana y preocupación de la población en general acerca de los problemas y las crisis.

11. La ciudadanía ejerce cada vez mayor presión sobre el gobierno de la ciudad y los funcionarios responsables. Por su parte, éstos actúan en situaciones inestables ${ }^{11}$ y con agendas sobrecargadas de problemas.

No pocos de estos aspectos tienen que ver con una cada vez más educada -es decir, politizada y consciente- ciudadanía, pendiente de su bienestar y del desempeño de los que administran el "hogar público". ${ }^{12}$ Pero una evaluación del desempeño de los gobiernos urbanos en países en desarrollo muestra, en general, que los funcionarios no tienen una orientación clara hacia el mejoramiento de las condiciones de bienestar de la población y de la calidad del medio físico urbano; su desempeño deja mucho que desear y muestra prácticas corruptas, agresivas e irresponsables, no sólo por lo anterior, sino también porque los recursos a su alcance son pocos y cuentan con poca preparación y bajos niveles de calificación; la burocracia urbana no está profesionalizada y el puesto se obtiene por recomendación o vínculos extraños; y la administración está sobrada en personal y es poco productiva, pero está, además, mal remunerada. ${ }^{13}$

${ }^{11}$ La inestabilidad propia de los gobiernos del mundo en desarrollo se refiere, además de a los acostumbrados golpes de estado, a la ausencia de procesos e instituciones que garanticen la alternancia en el poder, el llamado a cuentas, la permanencia de servidores públicos profesionales independientes del cambio de dirigentes políticos, etcétera.

${ }^{12}$ Como lo llama Daniel Bell (1977).

${ }^{13}$ Fried y Rabinowitz (1980) señalan éstas y otras características desde una perspectiva de análisis del desempeño administrativo y burocrático urbanos. 


\section{Los motivos}

El análisis político del proceso urbano y de las motivaciones de los agentes sociales se centra en la estructura y ejercicio del poder. En términos de estructura, ¿̇quién, en efecto, maneja, controla, gobierna la ciudad?, sería la pregunta inicial; y ¿cuál es la relación entre gobernantes y gobernados?, es la cuestión central relativa al ejercicio del poder, en el marco del control político y el cumplimiento de funciones en la gestión del proceso urbano.

Con relación al primer punto, y desde una perspectiva de sociología política, encontraríamos dos propuestas: aquella que señala que las ciudades son controladas por una estructura de poder "elitista", unificada, que opera tras bambalinas y es capaz de subordinar a sus propios intereses al aparato formal de gobierno; y, la segunda, que sugiere que el control sobre la ciudad se divide entre grupos diversos que compiten entre sí en un contexto "pluralista".

Es posible rechazar parcialmente la primera propuesta con base en resultados empíricos que señalan que el poder no se acumula o traslapa, y más bien se dispersa entre diferentes centros o medios de poder en un sistema complejo y multifuncional. Sin embargo, también es cierto que en ocasiones una élite adquiere y concentra tal poder que domina las discusiones cuando entran en juego sus intereses sectoriales en el ámbito local. Esto es especialmente válido en casos en donde el poder se ejerce para vetar una decisión o mantener el statu quo y preservar valores, mitos y procedimientos dominantes o establecidos. De esta manera se logra evitar que algunas cuestiones urgentes o importantes entren en la agenda política de los tomadores de decisiones.

\section{Población no organizada y agentes políticos}

Los anteriores aspectos afectan a aquellos -generalmente a la población pobre- que no tienen recursos suficientes para librar batallas políticas, administrativas o jurídicas. Esta situación se ha prestado para reflexionar sobre el papel de los planificadores o tomadores de decisiones y el de los ciudadanos no organizados o sin poder, en una situación de desigualdad no sólo económica sino también política. La respuesta podría estar en: 
1) la presencia de los partidos políticos, como una fuerza importante en la política urbana;

2) la figura poderosa (a veces paternalista y en ocasiones autoritaria) del gobernante;

3) la aparición de líderes cívicos (oportunistas o auténticos);

4) el fortalecimiento de organizaciones no gubernamentales o altruistas; $y$

5) el llamado planificador defensor, partidario de una causa, un grupo, un área o una zona (advocacy planner).

Todos estos agentes interpretan las necesidades de la población desorganizada de diversa forma, pero no siempre la traducen en demanda efectiva. Algunos sólo son capaces de movilizar a la población para protestar o respaldar alguna causa, pero no tienen interés o son incapaces de mantener la cohesión del grupo y, por tanto, no logran constituir un verdadero movimiento social.

La población desorganizada, sin embargo, encuentra en la arena de la política electoral una posibilidad de cambiar las cosas, es decir, su suerte; si bien como minoría estaría en desventaja y sus demandas pudieran no tener respuesta en este procedimiento que, por otro lado, permite, en principio, expresar preferencias y respaldar plataformas o posiciones políticas en el marco formal del proceso democrático. En el caso de México, las elecciones han resultado centrales a partir de los cambios iniciados con las reformas políticas y electorales después de las elecciones generales de 1988, cuando el partido dominante obtuvo en el D.F. menos de un tercio del total de votos para su candidato presidencial.

En otra escala, en el espacio comunitario, residencial, o vecinal, los problemas y las respuestas son diferentes. La capacidad organizativa, la demanda efectiva y la articulación de problemas, son posibles y han permitido, a una ciudadanía organizada, obtener respuesta de los gobiernos locales o urbanos que les ofrecen la posibilidad de compartir el control de los recursos. Esta relación entre gobernantes y gobernados puede tomar muchas formas, pero exige voluntad política y un esfuerzo gubernamental de descentralización, no sólo del control administrativo en la prestación y dotación de servicios públicos, sino también del control político del espacio urbano sobre el que se gobierna. 
En esta matriz formada por dos ejes: descentralización de funciones administrativas y descentralización del poder político, se obtienen al menos cuatro modelos de gobierno posibles: ${ }^{14}$

1) Modelo centralizado.

2) Modelo de gobierno comunitario.

3) Modelo de gobierno representativo.

4) Modelo burocrático.

Los modelos 2, 3 y 4 se relacionan con los tres aspectos que, según Castells ${ }^{15}$ son los que justifican una movilización política local: i) el conflicto sobre el consumo colectivo, con el 4; ii) el conflicto de la identidad cultural, con el 3; y iii) la demanda de autogestión local, con el 2. El 1 es el que ha estado presente durante el tiempo que persistió el nombramiento de regentes por parte del Ejecutivo Federal, cuando el Distrito Federal era un Departamento.

\section{Conclusiones}

El análisis propuesto de la relación entre gobernantes y gobernados y el interés por los resultados de la participación pública, ciudadana, en el ámbito de la política, deben permitir evaluar, por una parte, la capacidad de los ciudadanos como individuos, o bien como grupos organizados, para obtener respuesta a sus demandas o influir en las decisiones y acciones que lleva a cabo el Gobierno; y, por la otra, los logros que de la asignación de recursos o dotación de bienes y servicios por parte de la autoridad se obtienen en favor del bienestar de la población.

La posibilidad de influir, definir o determinar el proceso político a partir de la "voluntad popular", sería una buena definición teórica de democracia, pero en la práctica habría que contestar preguntas más prácticas y evaluar resultados concretos.

${ }^{14}$ La relación entre ambas formas de descentralización da lugar a cuatro posibilidades: 1) reducida descentralización del control público y de la prestación y manejo de servicios públicos; 2) una total descentralización en ambos casos, pasando por 3) servicios centralizados con una descentralización política, 4) o bien un manejo descentralizado de los servicios sin descentralizar el control político, como sería el caso de mantener u obtener representación en las instancias de decisiones o la posibilidad de decidir sobre la operación y el funcionamiento de un servicio, en los últimos dos casos, respectivamente.

${ }^{15}$ M. Castells, citado en Radisson, 1985. 
En síntesis, no sólo se trata de quién obtiene qué (recursos) y cuándo, sino también dónde, ${ }^{16}$ de la "bolsa pública".

La distancia entre teoría y práctica pudiera atribuirse, aunque como señalé antes no únicamente, a los problemas de escala entre las necesidades localizadas y la política y administración pública urbana. Esto se circunscribe al ámbito local, en donde es posible hacer coincidir las acciones públicas con las demandas efectivas de la comunidad por medio del activismo comunitario, y éste no sólo se inicia una vez que se toman decisiones (correctas o incorrectas, a favor o en contra) sino también, idealmente, desde la definición de los problemas y su incorporación a la agenda política.

Entonces, y para terminar:

1. El impacto o resultado de la asignación de recursos y dotación de bienes y servicios, en función de la capacidad comunitaria de influir en las decisiones y acciones públicas al respecto, puede ser evaluado o medido, y en todo caso fomentado o inducido, a partir de:

- la habilidad del activismo local para lograr que la asignación de recursos y la dotación de servicios públicos se hagan de acuerdo con la demanda colectiva (o individual), con base en la expresión de los deseos locales (o personales);

- el grado en que la descentralización impulsa el desarrollo de comunidades políticas locales; y

- el grado en que las acciones públicas tienen resultados redistributivos en términos políticos: redistribución del poder en favor de la comunidad local, y económicos: redistribución del ingreso real en favor de los pobres.

2. La capacidad de los ciudadanos para obtener respuesta a sus demandas e influir en las decisiones y acciones públicas será efectiva en función de que:

- el grupo organizado o activista sea capaz de articular sus objetivos en términos de demandas de bienes y servicios 
de consumo colectivo, cultura comunitaria y autogestión local;

- el grupo sea consciente de su papel como agente de cambio en la ciudad, independientemente de la escala o ámbito de su actuación;

- el grupo utilice correctamente a los agentes extralocales clave: los medios, los administradores, los profesionales, los partidos políticos, etcétera; y, finalmente,

- el grupo activista, si bien se ha ya conectado con el sistema político para fines prácticos, se separe ideológica y operativamente de un partido político determinado, es decir, mantenga su relativa autonomía.

\section{Bibliografía}

Bell, D. (1977), Las contradicciones culturales del capitalismo, México, CONACULTA.

Bowers, J. (1997), Sustainability and Environmental Economics, Longman.

Davidoff, P. (1965), “Advocacy and Pluralism in Planning”, JAIP, XXXI, (4).

De la Peña, G. (1998), "Políticas sociales, intermediación y participación popular en Guadalajara", Estudios Demográficos y Urbanos, 13 (2), núm. 38, pp. 407-426.

Fried, R. y F. Rabinowitz (1980), Comparative Urban Politics: A Performance Approach, Prentice Hall.

Graizbord, B. (1990), "Programa Nacional de Desarrollo Urbano 1990-1994: aspectos cualitativos y cuantitativos para una evaluación ex-ante", Estudios Demográficos y Urbanos, 5 (3), núm. 15, pp. 755-763.

(1989), “Zona Metropolitana de la Ciudad de México: fragmentación política y planeación del Valle CuautitlánTexcoco", en G. Garza, Una década de planeación urba- 
no-regional en México, 1978-1988, México, El Colegio de México, pp. 293-302.

Harvey, D. (1977), Urbanismo y desigualdad social, Madrid, Siglo XXI.

Hirschman, A. (1970), Exit, Voice and Loyalty, Harvard University Press.

Radisson, R. P. (1985), "Local Democracy in the city", en M. Pacione (ed.), Progress in Political Geography, Croom Helm.

Rodríguez, H. (1998), “Crecimiento demográfico y problemática ambiental en las áreas urbanas de Veracruz", en El Jarocho Verde, núm. 9, pp. 13-20.

Smith, D. (1980), Geografía Humana, Cop. Elementos de Geografía, Ed. Oikos-Tau, Barcelona.

Tamayo, S. (1997), "La participación ciudadana: un proceso", Revista Mexicana de Sociología, LIX (4), pp.155-185.

Yeates, D. (1977), The Ungovernable City, MIT. 
